\title{
An innovative ex-vivo porcine upper gastrointestinal model for submucosal tunnelling endoscopic resection (STER)
}

Authors

Institutions
Baldwin Yeung ${ }^{1}$, Philip Chiu ${ }^{1,2}$, Anthony Teoh ${ }^{1}$, Linfu Zheng ${ }^{2}$, Shannon Chan ${ }^{1}$, Kelvin Lam², Raymond Tang ${ }^{2}$, Enders K. W. Ng ${ }^{1,2}$

${ }^{1}$ Division of Upper GI \& Metabolic Surgery, Department of Surgery, the Chinese University of Hong Kong, Hong Kong, China ${ }^{2}$ Institute of Digestive Disease, the Chinese University of Hong Kong, Hong Kong, China submitted 12 . February 2016 accepted after revision 29. July 2016

\section{Bibliography Dol http://dx.doi.org/ 10.1055/s-0042-114980 Published online: 14.9.2016 Endoscopy International Open 2016; 04: E1101-E1106 (c) Georg Thieme Verlag KG Stuttgart · New York E-ISSN 2196-9736}

Corresponding author: Professor Philip Wai Yan Chiu, MD, FRCSEd, FHKAM

Department of Surgery Prince of Wales Hospital 32-34 Ngan Shing Street, Shatin Hong Kong

China

Fax: 85226377974

philipchiu@surgery.cuhk.edu.hk
Background and study aims: Submucosal tunnelling endoscopic resection (STER) is a novel endoscopic technique to remove submucosal tumour (SMT). We propose a novel, low cost simulator for training of techniques for STER.

Patients and methods: The model consisted of an ex-planted porcine oesophagus, stomach and duodenum with marbles embedded surgically in the submucosal plane. Two expert endoscopists with experience in submucosal tunnelling and 5 board-certified endoscopists with no experience in submucosal tunnelling were recruited. Participants were asked to perform a diagnostic endoscopy and 2 STER procedures, 1 in the oesophagus and 1 in the stomach. They also answered a structured questionnaire. Factors including operative time, mucosal and muscular injury rate, injection volume and accuracy of endoscopic closure were assessed.

Results: The median time for localization of all SMTs was 40.1 seconds for experts and $38.5 \mathrm{sec}-$ onds for novices $(P=1.000)$. For esophageal STER, the length of mucosal incisions and tunnelling distances were comparable between the 2 groups.

\section{Introduction}

Submucosal tunnelling endoscopic resection (STER) is a newly developed endoscopic technique for removal of gastrointestinal submucosal tumours such as small size gastrointestinal stromal tumour (GIST). A submucosal entry point is made typically $3 \mathrm{~cm}$ to $5 \mathrm{~cm}$ away from the target lesion. A submucosal tunnel is then created to access and remove the submucosal tumour. This technically challenging procedure has mainly been adopted in Asia.

The development of STER is in its infancy and case series have demonstrated some complications associated with this procedure. A Chinese case series reported the outcome of 48 patients with oesophageal submucosal tumours. The mean diam-
The median volume injected by the novice group was significantly lower than the experts $(15 \mathrm{~mL}$ vs $42.5 \mathrm{~mL}(P=0.05)$. The median tunnelling time per length was 25.9 seconds/mm for the experts and 40.8 seconds $/ \mathrm{mm}$ for the novice group $(P=0.38)$. There was a higher rate of mucosal injury and muscular perforation in the novice group ( 8 vs 0 ; $P=0.05$ ). For gastric STER, the length of mucosal incisions and tunnel distances were comparable between the 2 groups. The median tunnelling time per length for the experts was $23.3 \mathrm{sec}$ onds $/ \mathrm{mm}$ and 34.6 seconds $/ \mathrm{mm}$ for the novice group $(P=0.38)$. One mucosal injury was incurred by a novice. The rate of dissection in the stomach and the oesophagus was not statistically different $(P=0.620)$. All participants voted that the model provides a realistic simulation and recommended it for training.

Conclusions: STER is an advanced endoscopic technique where its indication is currently explored. Experienced and novice STER endoscopists have expressed the usefulness of this model as a training tool. This low-cost model can be used for future research in STER.

eter of the lesions was $22.9+/-12.1 \mathrm{~mm}$ and all patients had complete resection. Mean operative time was 41.8 minutes (range 15-140 minutes) and mean hospital stay was 2.4 days (range 1 day-13 days). It is of note that $10.4 \%$ of the patients had mucosal injury which required repair with endoscopic clips. Furthermore, $4.2 \%$ of the patients had submucosal tunnel infection which required re-closure of the submucosal tunnel entry point with clips. Pneumoperitoneum occurred in $4.2 \%$ of the patients and $6.3 \%$ had subcutaneous emphysema [1]. Another Chinese group reported 80 patients with GIST or leiomyoma treated by STER. It reported a mean operative time of 61.2 minutes and $97.6 \%$ complete resection rate. In this series, a lower rate of complication was observed. Complications observed in- 

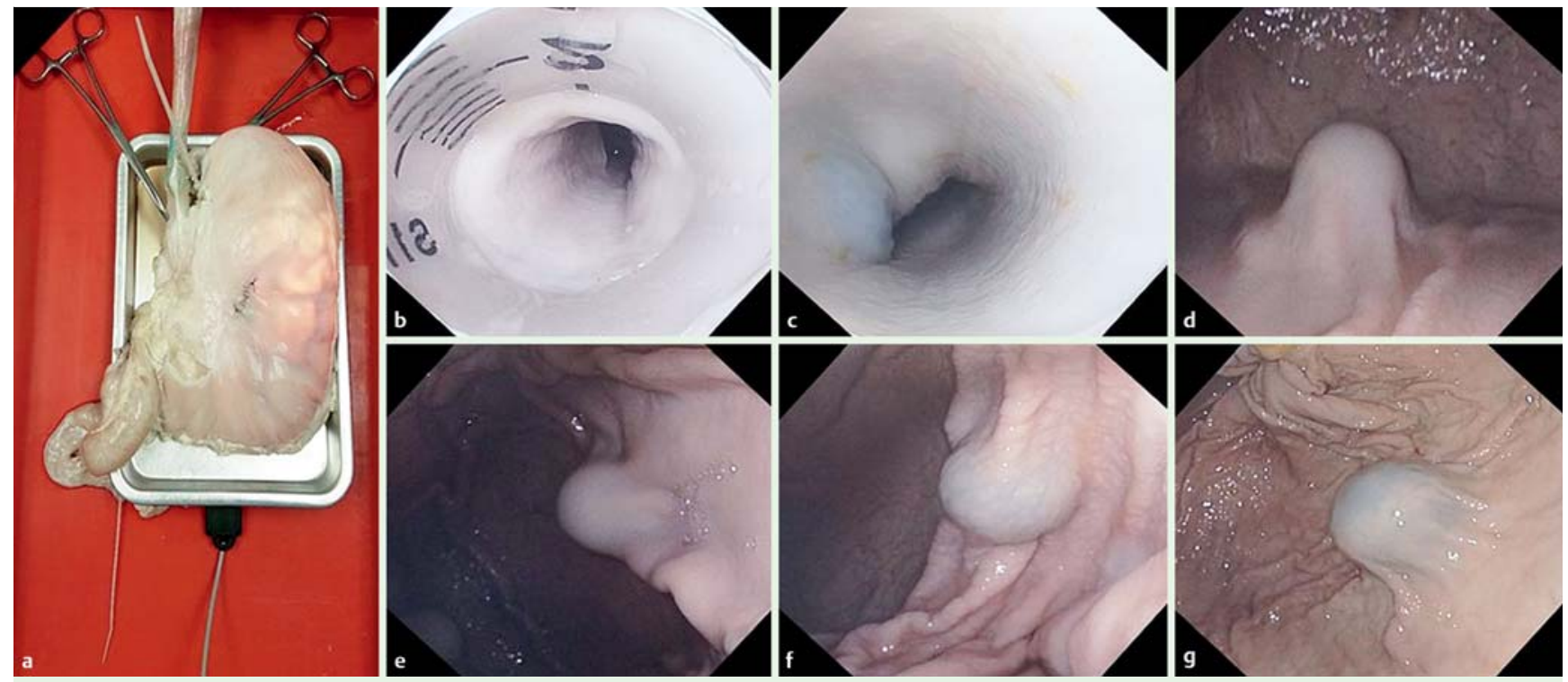

Fig. 1 a The STER model made of porcine esophagus, stomach and duodenum. b Endoscopic view of the entrance to the oesophagus. $\mathbf{c}$ Simulated submucosal tumour in the esophagus. $\mathbf{d}$ Simulated submucosal tumor in the posterior gastric body. e Simulated submucosal tumor in the anterior gastric body. f Simulated submucosal tumor in the antrum. g Simulated submucosal tumor in the gastric fundus.

cluded pneumothorax in $1.25 \%$ of patients which required chest drainage, mucosal tears in $1.25 \%$, subcutaneous emphysema in $2.5 \%$ and chest pain in $3.75 \%$ [2]. The technique is beginning to be adopted in the West [3]. The validity of this approach for the treatment of submucosal tumours remains to be confirmed with further follow up studies.

Simulation training is vital in order to produce competent surgeons and gastroenterologists capable of performing this advanced procedure. Currently, expensive computer simulators such as Simbionix GI mentor and the Accutouch system do not offer simulation for STER [4]. The ideal solution would be a lowcost, reproducible model which can be accessed in any endoscopy unit.

In this study, we will evaluate our novel model for training in the technique of submucosal tunnelling and endoscopic resection.

\section{Patients and methods}

$\nabla$

The model

The model is made of fresh porcine oesophagus, stomach and duodenum ( $\bullet$ Fig. 1). The duodenum was closed with a cable tie. Standardized $1.5-\mathrm{cm}$ glass marbles were used to simulate submucosal tumors. The marbles were placed in the submucosal space of the posterior aspect of the esophagus $5 \mathrm{~cm}$ from the esophagogastric junction, gastric fundus, anterior and posterior gastric body and gastric antrum. Each marble was placed in a small submucosal pocket through a small incision made in the seromuscular layer of the organ. The esophagus was then attached to a plastic simulated oropharynx using cable ties ( $\bullet$ Fig. 2) The simulated oropharynx was constructed using a hollow plastic tube (such as a 10 -mL plastic syringe with the injection end removed) covered by a pierced rubber diaphragm. A rubber diaphragm can easily be constructed using a rubber glove laid over the flanged end of the syringe and secured with a cable tie. The whole model was then laid on a metal tray. A diathermy pad was attached to the under surface of the metal tray to allow dissection with diathermy. This assembly was in turn placed into

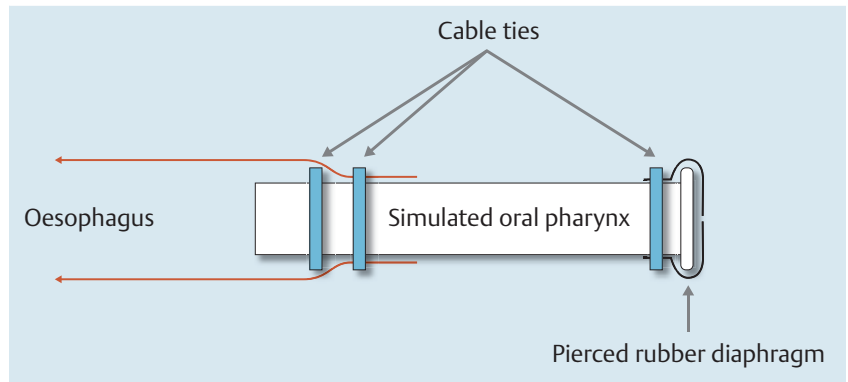

Fig.2 A method of creating a simulated oropharynx using a 10-mL plastic syringe, rubber glove, and 3 cable ties.

a water proof tray. The simulated oropharynx was secured against the edge of the water proof tray with a rubber sling. For improved stability and simulation of the hiatus, 2 metal artery forceps were clipped onto the serosa at the esophagogastric junction.

Five board-certified experienced endoscopists (each with more than 1000 endoscopic procedural experience but with no prior experience of endoscopic tunnelling) and 2 experts with significant experience in endoscopic tunnelling and STER have been recruited to participate in a once-off evaluation of our novel model.

Evaluation of the model was divided into 3 components. Each assessment was performed according to a written protocol. Each participant was asked to read the same set of written instruction carefully prior to assessment of the model. First, the endoscopist was asked to perform a diagnostic gastroscopy using a cap fitted gastroscope (Olympus EVIS EXERA III GIF-HQ190 gastroscope) with room air insufflation. A standard clear cap with venting channel was used. The perceived location of the tumor and the time of identification was recorded. Second, each endoscopist was asked to perform 2 STER procedures; 1 in the esophagus and 1 in the posterior gastric body. After submucosal injection with a solution of $1 \mathrm{~mL}$ indocarmine: $100 \mathrm{~mL}$ saline, the participants were asked to commence tunnelling $5 \mathrm{~cm}$ away from the 


\section{Questionnaire}

1. Is the STER simulator easy to use?

\begin{tabular}{|l|l|l|l|l} 
Very Easy & Easy & Average & Difficult & Very Difficult \\
\hline
\end{tabular}

\section{Is the STER simulator useful for training residents?}

\begin{tabular}{|c|l|l|l|l|}
\hline Strongly Agree & Agree & Neither & Disagree & $\begin{array}{c}\text { Strongly } \\
\text { Disagree }\end{array}$ \\
\hline
\end{tabular}

3. If you have used other endoscopy simulators, is the STER simulator better or worse than the other simulators you have used?

Better Equivalent

Worse

\section{Please state which simulators you have used before}

4. Do you recommend using the STER simulator for future training?

\begin{tabular}{|c|c|c|c|c|}
\hline Strongly Agree & Agree & Neither & Disagree & $\begin{array}{c}\text { Strongly } \\
\text { Disagree }\end{array}$ \\
\hline
\end{tabular}

5. Is the STER simulator an appropriate testing format for trainees prior to performance of STER in patients?

\begin{tabular}{l|l|l|l|l} 
Yes & & & & No
\end{tabular}

\section{How can we improve our simulator?}

target lesion through a longitudinal mucosal incision using a TT knife supported by an ERBE electrosurgery unit. The time from incision to complete freeing of the submucosal marble was recorded. The participants were not required to remove the marble from the submucosal space. The participants were asked to close the mucosal defect using endoscopic clips (Olympus QuickClip Pro). Closure time was recorded. Other variables such as tunnel length, mucosal defect length, clips apposition accuracy, and volume of submucosal injection were also recorded. Third, the participants were asked to fill in a short questionnaire to ascertain their subjective assessment of the STER model. The questionnaire assessed the participants' perception of the model's ease of use and its usefulness as a training tool ( $\bullet$ Fig.3) It also ascertained their opinion as to whether it will be useful for training and testing future residents, as well as how the model compares to the participant's past experience with other simulators. Statistical analysis was performed using SPSS software. Analysis was made using the Mann-Whitney $U$ test and z-proportion test Statistical significance was assessed at $P<0.05$.
Fig.3 Questionnaire to obtain subjective evaluation of the STER model. 

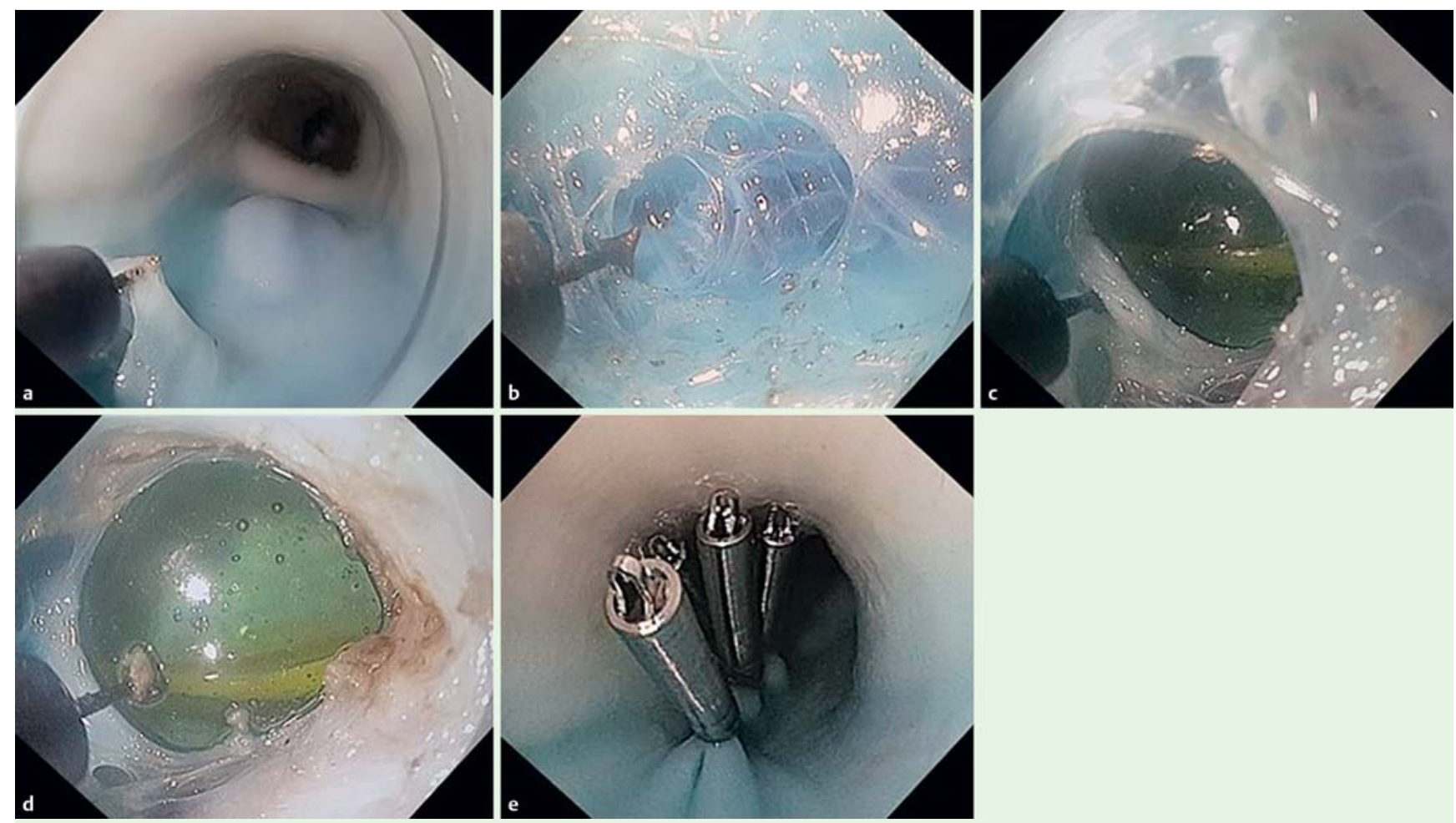

Fig. 4 Endoscopic views during the performance of submucosal tunnelling in the STER model. a Creation of entrance to submucosal tunnel. b, c, d Submucosal tunnelling toward the simulated submucosal tumor. e Closure of mucosal defect with endoscopic clips.

$(P=0.38)$. One candidate in the novice group abandoned procedure due to failure to progress. The median closure time (adjusted for the length of the mucosal defect) for the expert group was 11 seconds/mm and 15 seconds $/ \mathrm{mm}$ for the novice group $(P=$ 0.38 ). Both groups achieved complete clip apposition. No tunnel mucosal tear was incurred by the expert group. However, 1 tunnel mucosal tear was incurred in the novice group. A total of 7 muscle tears were incurred by the novice group and no muscle tears were incurred by the expert group $(P=0.05)$. All visualized muscle tears were separate from the seromuscular incision use for placement of simulated submucosal tumour ( $\bullet$ Table 1 ). The proportion of successful mucosal closure clip placement was not significantly different between experts and novices $(P=0.267)$.

\section{Stomach}

The median volume injected by the novice group was $28.5 \mathrm{~mL}$. The median volume injected by the expert group was $42.5 \mathrm{~mL}$ $(P=0.06)$. The median tunnel length for the expert group was $31 \mathrm{~mm}$ and $30 \mathrm{~mm}$ for the novice group $(P=1.00)$. The median mucosal defect for the expert group was $33.5 \mathrm{~mm}$ and $26 \mathrm{~mm}$ for the novice group $(P=0.85)$. The median tunnelling time (adjusted for the distance of dissection) for the expert group was 23.3 seconds $/ \mathrm{mm}$ and 34.6 seconds/mm for the novice group $(P=0.38)$. The median closure time (adjusted for the length of the mucosal defect) for the expert group was 9.7 seconds $/ \mathrm{mm}$ and 18.2 seconds $/ \mathrm{mm}$ for the novice group $(P=0.38)$. Only 1 tunnel mucosal tear was incurred in the novice group ( $\bullet$ Table 2 ). Statistical comparison of the tunnelling time per tunnel length $(\mathrm{mm})$ between the stomach and the esophagus was performed. This showed that there was no significant difference between the $2(P=0.620)$. The proportion of successful mucosal clip placement was not significantly different between experts and novices $(P=0.116)$.

\section{Task 3: Questionnaire assessment}

All participants suggested that STER simulator provided a realistic model of submucosal tumours and strongly recommended that the STER simulator be incorporated into formal training and testing endoscopist preparing to perform submucosal tunnelling. Four of the participants have had experience with other computer-based simulators and thought the STER model was a more accurate reflection of real life endoscopic experience. However, 3 of the 5 novice participants thought that the STER model was difficult to use while the remaining participants thought the model was easy to use. Suggested improvements include improvement in the stability of the oesophagus and stomach, as well as use of a thickener to improve submucosal injection retention time.

\section{Discussion}

$\nabla$

Historically, the management of submucosal mass was either surgery or active observation. The American Gastroenterological Association Institute recommends that lesions such as lipoma, duplication cysts, pancreatic rest, inflammatory fibroid polyp and Schwannoma do not require investigation or follow up. For gastrointestinal stromal tumor (GIST), an arbitrary cut off of 3 $\mathrm{cm}$ has been recommended. It has been recommended that GIST lesser than $3 \mathrm{~cm}$ should be actively observed while those greater than $3 \mathrm{~cm}$ should be surgically resected [5]. The development of new, effective and safe minimally invasive techniques will alter such treatment guidelines.

Endoscopic submucosal excavation of a submucosal lesion through an incision directly over the lesion have been attempted before $[6,7]$. However, direct excavation of the tumour arising from the muscularis propria risks a high chance of full thickness perforation. A mucosal tunnel may act as a safety valve that will 
Table 1 Outcomes for esophageal submucosal tunnelling.

$\begin{array}{rrrrrrrrrrr}\text { Novice } & 3 & 43.2 & 23 & 27 & 0 & 1 & 18.9 & 100 \% & 10 \\ & 4 & 91.7 & 15 & 12 & 0 & 2 & 26.0 & 100 \% & 20 \\ & 5 & 40.8 & 20 & 30 & 1 & 2 & 15.0 & 50 \% & 20 \\ & 6 & 29.9 & 25 & 10 & 0 & 1 & 10.4 & 100 \% & 15 \\ & 7 & 24.7 & 30 & 5 & 1 & 1 & 12.6 & 100 \%\end{array}$

Table 2 Outcomes for gastric submucosal tunnelling.

\begin{tabular}{|c|c|c|c|c|c|c|c|c|c|}
\hline & Participant & $\begin{array}{l}\text { Tunnelling } \\
\text { time (s) per } \\
\text { mm dissection }\end{array}$ & $\begin{array}{l}\text { Mucosal } \\
\text { defect } \\
(\mathrm{mm})\end{array}$ & $\begin{array}{l}\text { Tunnel } \\
\text { length } \\
(\mathrm{mm})\end{array}$ & $\begin{array}{l}\text { Mucosal } \\
\text { tear }\end{array}$ & $\begin{array}{l}\text { Muscle } \\
\text { tear }\end{array}$ & $\begin{array}{l}\text { Closure time } \\
\text { (s) per mm } \\
\text { defect }\end{array}$ & $\begin{array}{l}\text { \% of clips with } \\
\text { good mucosal } \\
\text { apposition }\end{array}$ & $\begin{array}{l}\text { Volume } \\
\text { injected } \\
(\mathrm{mL})\end{array}$ \\
\hline \multirow[t]{2}{*}{ Expert } & 1 & 16.4 & 20 & 35 & 0 & 0 & 6 & $100 \%$ & 35 \\
\hline & 2 & 30.2 & 47 & 27 & 0 & 0 & 13.4 & $92 \%$ & 50 \\
\hline \multirow[t]{5}{*}{ Novice } & 3 & 21.6 & 26 & 24 & 0 & 0 & 15.0 & $100 \%$ & 25 \\
\hline & 4 & 55.2 & 20 & 30 & 0 & 0 & 40.5 & $50 \%$ & 30 \\
\hline & 5 & 26.6 & 20 & 45 & 0 & 0 & 25.3 & $29 \%$ & 27 \\
\hline & 6 & 37.6 & 65 & 50 & 0 & 0 & 4.6 & $67 \%$ & 30 \\
\hline & 7 & 34.6 & 60 & 10 & 1 & 0 & 18.2 & $82 \%$ & 76 \\
\hline
\end{tabular}

minimize the risk of full-thickness perforation [8]. Mucosal tunnelling has been successfully applied to the treatment of achalasia in form of per oral endoscopic myotomy (POEM) [9]. Inspired by this technique, preliminary experiences of submucosal tumour excision through a submucosal tunnel were published since 2012 [10-13]. Since then, larger case series of STER have been reported $[14,15]$. With the development and validation of STER as a minimally invasive technique for submucosal tumor removal, it can be envisioned that small size GIST can be excised endoscopically. This option may prove to be more readily accepted by patients as well as more cost effective. In order for this to be practical, an effective training methodology is vital for the propagation of this novel technique.

STER is a very difficult technique to master. The purpose of the current study is to objectively assess the usefulness of a bench top model in training STER as perceived by busy clinicians. It is not intended to be a study to assess the learning curve required for STER nor is it designed to assess the technical challenges of performing STER in different locations within the stomach. Such assessment will be the basis of future studies. As such, each endoscopists were only asked to perform 2 STER procedures. The posterior gastric body was chosen as it is the easiest location to perform STER in the stomach. To our knowledge, there is yet no formal validated model for STER training. In this study, we offer the first report on a training model for STER. It is simple and low cost. In contrast to other porcine per oral endoscopic myotomy (POEM) training model, our model trains individuals to perform directional submucosal tunnelling [16]. This is especially useful in the stomach where one can easily get lost within the submucosal space. Furthermore, unlike the other model which places the model on a corkboard, our design uses a metal tray thereby permitting the usage of diathermy. This further enhances the dissection experience.

All participants have agreed that the endoscopic appearance of the simulated submucosal tumours reflects real life experience. We recognize that the submucosal marble is non adherent to the muscularis propria. In this aspect, it is dissimilar to that of real submucosal tumours. Also, it may be easier to perform submucosal tunnelling in the porcine model as the mucosa is much tougher than that of human. Furthermore, it does not simulate active bleeding. Hemostasis can be very difficult when the operative field is limited to the submucosal plane. In theory, gastric STER should be more challenging than esophageal STER due to the technical challenge of creating a submucosal tunnel in the expanse of the gastric lumen. The rate of tunnelling per se should not be different in the stomach or the oesophagus. This is confirmed in our data. We believe that is the reason that the rate of tunnelling was no different between gastric and oesophageal tunnelling. Despite these shortcomings, the model has enabled participants to appreciate the importance of good placement of mucosal entry point, the difficulty in mucosal tunnel entry, and especially in the stomach, the risk of making navigational error and missing the target lesion. Moreover, all participants have recommended that this model to be incorporated into the training and assessment of future STER practitioners.

There are several limitations to the current study. First, the number of participants is small. Globally, STER remains the remit of only a handful of advanced endoscopists. It is therefore very difficult to recruit a large number of expert endoscopists who has STER as part of their repertoire. Therefore, we have only been able to include two expert endoscopists in this study. Furthermore, we limited participants to busy clinicians with significant endoscopic experience. This is so that the evaluation cohort will mimic the future users of the STER model. The statistical power of the study is therefore limited by the relatively small number of assessors. Interestingly, the procedural speed did not significantly differ between the novices and the experts. This may reflect that although the novices have no experience with the technique, they are all experienced endoscopists who are working in a regional referral centre with a STER service. It maybe that they are very familiar with the principles and practicalities of submucosal tunnelling. The intention of the study was not to assess the learning curve of STER. This study has not been able to identify the normal learning curve of STER procedure. Although the differences procedural speed between the novices and experts did not reach statistical significance, the most important objective that a training model should achieve is that it is able to accurately simulate the occurrence of significant complications. In this study, we observed that there was a significantly higher number of muscle injury incurred by the novice group during oesophageal tunnel- 
ling. These muscle injuries were clearly separate from the pre-existing seromuscular incision used for simulated SMT placement. As such, our model has demonstrated its ability to differentiate between novices and experts in terms of complication rate.

Three of the novices in this study found that the STER model was difficult to use. It has been well documented that entry into the submucosal tunnel is the most difficult step of an endoscopic tunnelling procedure. The perceived difficulty in using the STER model may simply be a reflection of STER being a very difficult procedure to perform. Most participants suggested that the stability of the esophagus and the stomach can be improved. The incorporation of a grooved tray similar to the Erlangen Compact EASIE simulator $[17,18]$ may improve organ stability. Currently, glass marbles with blue coloring were used to simulate SMT. A SMT made of an opaque white material can be used in the future to further mimic the appearance of SMT.

\section{Conclusions}

\section{$\nabla$}

STER is an advanced endoscopic technique where its indication is currently being actively explored. An effective bench top model will be an important part of education and training for endoscopists wishing to add STER to their repertoire. We have presented a simple and low cost bench top model for STER training. Experienced and novice STER endoscopists have expressed the usefulness of this model. Further learning curve study will enhance the validity of this approach in training STER.

\section{Competing interests: None}

\section{References}

1 Zhao $H$, Sheng $H$, Huang $L$ et al. Submucosal tunnelling endoscopic resection in the treatment of esophageal submucosal tumours originating from muscularis propria layer. Zhonghua Wei Chang Wai Ke Za Zhi 2015; 18: $478-482$

2 Wang H, Tan Y, Zhou Y et al. Submucosal tunnelling endoscopic resection for upper gastrointestinal submucosal tumours originating from the muscularis propria layer. Eur J Gastroenterol Hepatol 2015; 27: $776-780$
3 Reinehr R. Endoscopic submucosal excavation (ESE) is a safe and useful technique for endoscopic removal of submucosal tumours of the stomach and the oesophagus in selected cases. Z Gastroenterol 2015; 53: $573-578$

4 Bar-Meir S. Simbionix simulator. Gastrointest Endosc Clin N Am 2006; 16: $471-478$

5 American Gastroenterological Association. Institute medical position statement on the management of gastric subepithelial masses. Gastroenterology 2006; 130: 2215-2216

6 Lee IL, Lin PY, Tung SY et al. Endoscopic submucosal dissection for the treatment of intraluminal gastric subepithelial tumours originating from the muscularis propria layer. Endoscopy 2006; 38: 1024-1028

7 Hwang JC, Kim JH, Kim JH et al. Endoscopic resection for the treatment of gastric subepithelial tumours originated from the muscularis propria layer. Hepatogastroenterology 2009; 56: 1281 - 1286

8 Sumiyama K, Gostout CJ, Rajan E et al. Submucosal endoscopy with mucosal flap safety valve. Gastrointest Endosc 2007; 65: 688-694

9 Inoue H, Minami H, Kobayashi $Y$ et al. Peroral endoscopic myotomy (POEM) for esophageal achalasia. Endoscopy 2010; 42: 265 - 271

$10 \mathrm{Xu}$ MD, Cai MY, Zhou PH et al. Submucosal tunnelling endoscopic resection: a new technique for treating upper GI submucosal tumours originating from the muscularis propria layer (with videos). Gastrointestinal Endoscopy 2012; 75: 195 - 199

11 Lee CK, Lee SH, Chung IK et al. Endoscopic full-thickness resection of a gastric subepithelial tumor by using the submucosal tunnel technique with the patient under conscious sedation (with video). Gastrointest Endosc 2012; 75: 457 - 459

12 Inoue $H$, Ikeda $H$, Hosoya $T$ et al. Submucosal endoscopic tumor resection for subepithelial tumors in the esophagus and cardia. Endoscopy 2012; 44: 225 - 230

13 Gong W, Xiong Y, Zhi F et al. Preliminary experience of endoscopic submucosal tunnel dissection for upper gastrointestinal subepithelial tumours. Surg Endosc 2014; 28: 524 - 530

14 Ye LP, Zhang Y, Mao XL et al. Submucosal tunnelling endoscopic resection for small upper gastrointestinal subepithelial tumours originating from the muscularis propria layer. Surg Endosc 2014; 28: 524 - 530

15 Wang $L$, Ren W, Zhang $Z$ et al. Retrospective study of endoscopic submucosal tunnel dissection (ESTD) for surgical resection of esophageal leiomyoma. Surg Endosc 2013; 27: 4259-4266

16 Clark $W$, Velanovich $V$. Porcine esophageal-gastric explants: a feasible training model for peroral endoscopic myotomy (POEM). Abstract Mo2074. SAGES conference 2012

17 Hochberger J, Neumann $M$, Hohenberger Wet al. New endoscopy trainer for flexible therapeutic endoscopy [German]. Z Gastroenterol 1997; 35: $722-723$

18 Hochberger J, Maiss J. Currently available simulators: ex vivo models. Gastrointest Endosc Clin N Am 2006; 16: 435-439 